\title{
Baroclinic Instability of Nonzonal Flows and Bottom Slope Effects on the Propagation of the Most Unstable Wave
}

\author{
HENGLING LENG \\ College of Oceanography, Hohai University, Nanjing, China \\ XUEZHI BAI \\ College of Oceanography, and International Polar Environment Research Laboratory, \\ Hohai University, Nanjing, China
}

(Manuscript received 30 April 2018, in final form 8 October 2018)

\begin{abstract}
A linear, two-layer potential vorticity (PV) equation model on the $\beta$ plane is employed to study the baroclinic instability of nonzonal basic currents flowing over a uniform bottom slope. Criteria for the instability, phase speed, and growth rate of unstable waves are all given as functions of the basic shear velocity, $\beta$, and bottom slope. The study suggests two kinds of long wave cutoff, one induced by the slope and the other by $\beta$; the first one exists in all directions, while the second requires at least a slight deviation of the wave vector from the meridians. Subtle differences between configurations of the PV gradient lead to completely different characteristics of unstable perturbations, such as propagation and scale. In the case of a positive slope (the bottom slope in the same direction as the isopycnal tilt), the fastest-growing wave is capable of propagating across the basic flow streamlines. By contrast, in the case of a negative slope (the bottom slope opposed to the isopycnal tilt), the most unstable wave always propagates along the streamlines. In addition, the spatial scale of the most unstable mode can be heavily reduced by a negative slope.
\end{abstract}

\section{Introduction}

Classic baroclinic instability theory was developed by Charney (1947) and Eady (1949) to explain the general character of observed fluctuations in the atmosphere. The consideration of stratification and baroclinicity in their models allows the presence of available potential energy, which could be transferred to perturbation energy as the basic flow or initial disturbance achieves a certain criterion. Phillips (1954) introduced a simpler two-layer model that filtered out disturbances with small vertical scales. Pedlosky (1987) applied Phillips' model to diagnose the nonzonal basic flows over a flat bottom and noted that such flows would not possess a minimum critical shear for instability. It was further found that even slight nonzonality in the mean flow would easily radiate perturbation energy to the far field (Kamenkovich and Pedlosky 1996; Hristova et al. 2008). Namely, nonzonal flows are more unstable than purely zonal ones (Rhines 1977; Spall 2000; Smith 2007).

\footnotetext{
Corresponding author: Xuezhi Bai, xuezhi.bai@hhu.edu.cn
}

In the real ocean, relatively stable nonzonal currents are common because of the presence of lateral boundaries or bottom slopes. A bottom slope usually helps stabilize the basic flow by modifying the background PV gradient, which is quite similar to the $\beta$ effect (Blumsack and Gierasch 1972; Steinsaltz 1987; Chen and Kamenkovich 2013). However, the slope can also destabilize the flow when the topography reaches to a large amplitude relative to the total depth (Orlanski 1969; Tanaka and Akitomo 2001). Mysak (1977) and Ikeda (1983) constructed a two- and threelayer model on the $f$ plane to analyze the instability of the currents flowing over a sloping bottom. They found the slope heavily affects the instability scale. Wang and Ikeda (1997) diagnosed the growth rate of unstable waves with flat, gentle, medium, and steep slopes by means of numerical experiments, verifying that a positive slope can result in a lower wavenumber (larger scale) of the most unstable wave, while a gentle negative slope favors the wave with the maximum growth rate shifting toward a higher wavenumber (smaller scale). 
Many studies suggested that the eddies or meanders near the continent slope are produced by local instability (Gill et al. 1974; McWilliams et al. 1983; Xue and Mellor 1993; Kurian et al. 2011). The propagation direction of the most unstable wave is important in understanding the initial track of mesoscale eddies or water exchanges between the basic flow and the surrounding area. However, the propagation direction was seldom involved in previous studies primarily due to the "channel" configuration: unstable waves default to travel parallel to the lateral boundaries (e.g., Charney 1947; Eady 1949; Phillips 1954; Wang and Ikeda 1997). In addition, the deviation of the most unstable wave from the basic flow streamlines is hardly noticeable without consideration of the combined effect of $\beta$ and bottom slope.

In this study, the nonzonality, $\beta$, and bottom slope were taken into account simultaneously. To investigate the slope effects on propagation directions of unstable waves, lateral boundaries were removed. We also discussed the dependences of long wave cutoff and instability scale on the slope or $\beta$, in comparison with the results of purely zonal flows (Blumsack and Gierasch 1972; Mysak 1977; Ikeda 1983; Wang and Ikeda 1997), and meridional flows (Walker and Pedlosky 2002).

The paper is organized as follows: section 2 describes a two-layer model of nonzonal basic currents flowing over a uniform bottom slope. Section 3 gives the dispersion relation, phase speed, and growth rate of unstable waves. In section 4, slope effects on wave propagations and scales are discussed. Finally, in section 5, the conclusions are summarized.

\section{The model}

A two-layer potential vorticity (PV) equation model of nonzonal inviscid currents was derived by Pedlosky (1987) on the basis of the quasigeostrophic theory. Now, we introduce the topographic term in the equation and assume that the basic flow is flowing over a bottom slope (Fig. 1). Lateral boundaries and horizontal velocity shear are both ignored in order to focus on the effects of bottom slope and baroclinicity. The equations of $\mathrm{PV}$ are given by

$$
\begin{aligned}
& \left(\frac{\partial}{\partial t}+u_{1} \frac{\partial}{\partial x}+v_{1} \frac{\partial}{\partial y}\right) q_{1}=\frac{\operatorname{curl}_{z} \tau}{\rho_{1} H_{1}} \\
& \left(\frac{\partial}{\partial t}+u_{2} \frac{\partial}{\partial x}+v_{2} \frac{\partial}{\partial y}\right) q_{2}=0
\end{aligned}
$$

where the subscripts 1 and 2 refer to quantities in the upper and lower layer, respectively; $u_{n}$ and

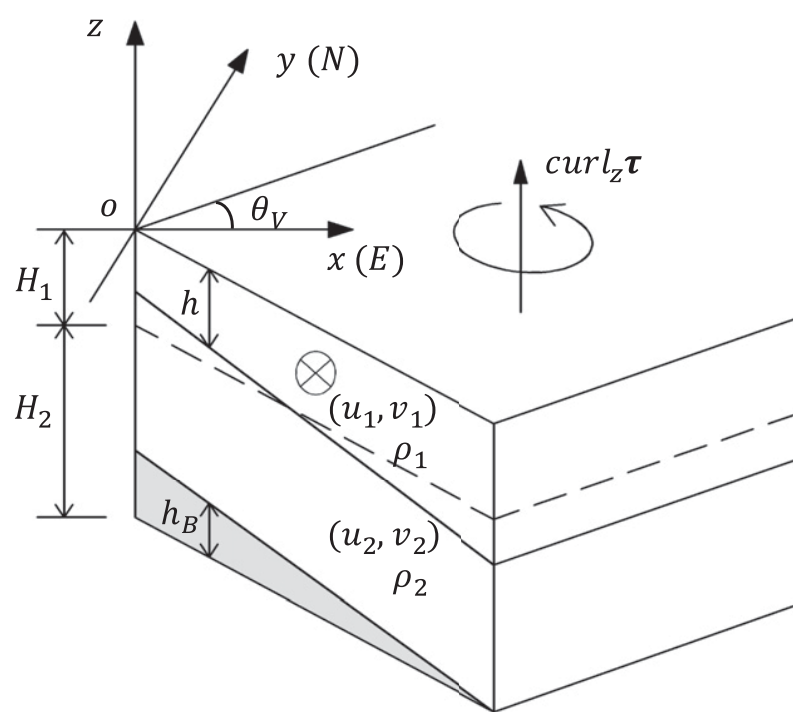

FIG. 1. Two-layer model of basic nonzonal flows over a bottom slope for instability analysis: $u_{n}, v_{n}(n=1,2)$ are components of velocity, the density $\rho_{n}$ and the average depth $H_{n}$ are constant in each layer, $h$ is the spatially variable depth of the thermocline, $h_{B}$ is the bottom topography, and the curl of surface wind stress is denoted by $\operatorname{curl}_{z} \tau$.

$v_{n}(n=1,2)$ are components of velocity; and $q_{n}$ represents PV. The density $\rho_{n}$ and the average depth $H_{n}$ are constant in each layer. As an external force, the curl of surface wind stress denoted by $\operatorname{curl}_{z} \tau$ only acts on the equilibrium of $q_{1}$.

The streamfunction $\psi_{n}$ is introduced, and $q_{n}$ is of the form

$$
\begin{aligned}
& q_{1}=\nabla^{2} \psi_{1}+f+F_{1}\left(\psi_{2}-\psi_{1}\right), \\
& q_{2}=\nabla^{2} \psi_{2}+f+\frac{f_{0}}{H_{2}} h_{B}-F_{2}\left(\psi_{2}-\psi_{1}\right),
\end{aligned}
$$

where $\nabla^{2} \psi_{n}$ is the relative vorticity, $f$ is the planetary vorticity, $h_{B}$ is the bottom topography, and $F_{n}\left(\psi_{2}-\psi_{1}\right)$ the stretching term. Under the $\beta$-plane approximation, $f=f_{0}+\beta y, f_{0}$ is the Coriolis parameter and $\beta$ is the planetary vorticity gradient. With the reference density $\rho_{0}$ and the reduced gravity $g^{\prime}=g\left(\rho_{2}-\rho_{1}\right) / \rho_{0}, F_{n}$ is defined as $F_{n}=f_{0}^{2} /\left(g^{\prime} H_{n}\right)$, square of the inverse Rossby radius.

Suppose that streamfunction $\psi_{n}$ can be written as a combination of the basic part and small perturbation: $\psi_{n}=\Psi_{n}+\phi_{n}$. It is appropriate to set $\Psi_{1}=-U y+V x$ and $\Psi_{2}=0$ so that the value of $|\mathbf{V}|=\left(U^{2}+V^{2}\right)^{1 / 2}$ describes the basic shear velocity. The perturbation equations for $\phi_{n}$ are obtained by linearizing Eqs. (2.1a) and (2.1b): 


$$
\begin{aligned}
&\left(\frac{\partial}{\partial t}\right.\left.+U \frac{\partial}{\partial x}+V \frac{\partial}{\partial y}\right)\left[\nabla^{2} \phi_{1}+F_{1}\left(\phi_{2}-\phi_{1}\right)\right] \\
&+\left(\beta+F_{1} U\right) \frac{\partial \phi_{1}}{\partial x}+F_{1} V \frac{\partial \phi_{1}}{\partial y}+\beta V=\frac{\operatorname{curl}_{z} \tau}{\rho_{1} H_{1}}, \\
& \frac{\partial}{\partial t}\left[\nabla^{2} \phi_{2}-F_{2}\left(\phi_{2}-\phi_{1}\right)\right]+\left(\beta+\frac{f_{0}}{H_{2}} \frac{\partial h_{B}}{\partial y}-F_{2} U\right) \frac{\partial \phi_{2}}{\partial x} \\
& \quad-\left(\frac{f_{0}}{H_{2}} \frac{\partial h_{B}}{\partial x}+F_{2} V\right) \frac{\partial \phi_{2}}{\partial y}=0,
\end{aligned}
$$

where the presence of meridional transport requires

$$
\beta V=\frac{\operatorname{curl}_{z} \tau}{\rho_{1} H_{1}},
$$

the Sverdrup relation. This balance mechanism is merely a choice to simplify Eq. (2.2a) and to obtain homogenous equations for $\phi_{n}$ (Pedlosky 1987; Arbic 2000).

\section{Identification of baroclinic unstable modes}

We seek planetary wave solutions of the form

$$
\phi_{n}=A_{n} e^{i(k x+l y-\sigma t)},
$$

where $A_{n}$ is the amplitude in each layer, $(k, l)$ is the wavenumber, and $\sigma$ is the circular frequency. Substitution of Eq. (3.1) into Eqs. (2.2a) and (2.2b) yields two coupled algebraic equations for $A_{n}$,

$$
\begin{aligned}
& {\left[\sigma\left(K^{2}+F_{1}\right)+k \beta-K^{2}(k U+l V)\right] A_{1}} \\
& \quad-(\sigma-k U-l V) F_{1} A_{2}=0, \\
& -\sigma F_{2} A_{1}+\left[\sigma\left(K^{2}+F_{2}\right)+k \beta-F_{2}(k U+l V)\right. \\
& \quad+k \partial B / \partial y-l \partial B / \partial x] A_{2}=0,
\end{aligned}
$$

where $K^{2}=k^{2}+l^{2}$ and $B=f_{0} h_{B} / H_{2}$.

\section{a. The dispersion relation}

In the local Cartesian frame, the variable directions of $\mathbf{V}$ and $\nabla B$, where $\theta_{V}, \theta_{B} \in[0,2 \pi)$, may cause difficulties in further analysis. Thus, a wavenumber coordinate system is introduced (Fig. 2), where the unit vectors $\mathbf{e}_{1}$ and $\mathbf{e}_{2}$ are set separately in the tangential and normal direction of wave vector $\mathbf{K}$ ( $\mathbf{K}$ crosses the $x$ axis at an arbitrary angle $\theta$ ). The problem is greatly simplified by using the following definitions:

$$
\begin{aligned}
& \tilde{U}=\frac{\mathbf{K} \cdot \mathbf{V}}{K}=\frac{k U+l V}{K}=|\mathbf{V}| \cos \left(\theta_{V}-\theta\right), \\
& \hat{\beta}=\frac{\mathbf{K} \times \nabla f}{K}=\frac{k \beta}{K}=\beta \cos \theta, \\
& \hat{S}=\frac{\mathbf{K} \times \nabla B}{K}=\frac{k \partial B / \partial y-l \partial B / \partial x}{K}=|\nabla B| \sin \left(\theta_{B}-\theta\right),
\end{aligned}
$$

where $\tilde{U}$ is the projection of $\mathbf{V}$ on $\mathbf{e}_{1}$ and $\hat{\beta}$ and $\hat{S}$ are projections of $\nabla f$ and $\nabla B$ on $\mathbf{e}_{2}$, respectively. In matrix form, Eqs. (3.2a) and (3.2b) become

$$
\left[\begin{array}{cc}
\sigma\left(K^{2}+F_{1}\right)+K \hat{\beta}-K^{3} \tilde{U} & -(\sigma-K \tilde{U}) F_{1} \\
-\sigma F_{2} & \sigma\left(K^{2}+F_{2}\right)+K \hat{\beta}+K \hat{S}-F_{2} K \tilde{U}
\end{array}\right]\left[\begin{array}{l}
A_{1} \\
A_{2}
\end{array}\right]=0
$$

Nontrivial solutions for $A_{1}$ and $A_{2}$ can be found only if the determinant of the coefficient matrix vanishes. This condition yields a quadratic equation for $\sigma$,

$$
\begin{aligned}
& K^{2}\left(K^{2}+F_{1}+F_{2}\right) \sigma^{2}+K\left[-K^{2}\left(K^{2}+2 F_{2}\right) \tilde{U}+\left(2 K^{2}+F_{1}+F_{2}\right) \hat{\beta}+\left(K^{2}+F_{1}\right) \hat{S}\right] \sigma \\
& \quad+K^{2}\left(-K^{2} \tilde{U}+\hat{\beta}\right)\left(-F_{2} \tilde{U}+\hat{\beta}+\hat{S}\right)=0,
\end{aligned}
$$

the dispersion relation.

b. Phase speed and growth rate of unstable modes

The discriminant of $\sigma$ derived from Eq. (3.4) is

$$
\begin{aligned}
\Delta= & -K^{4}\left(4 F_{1} F_{2}-K^{4}\right) \tilde{U}^{2}+\left(F_{1}+F_{2}\right)^{2} \hat{\beta}^{2}+\left(F_{1}+K^{2}\right)^{2} \hat{S}^{2}+2 K^{4}\left(F_{1}-F_{2}\right) \tilde{U} \hat{\beta} \\
& +2 K^{2}\left(K^{4}+F_{1} K^{2}-2 F_{1} F_{2}\right) \tilde{U} \hat{S}+\left[2 K^{2}\left(F_{1}-F_{2}\right)+F_{1}^{2}+F_{2}^{2}\right] \hat{\beta} \hat{S} .
\end{aligned}
$$




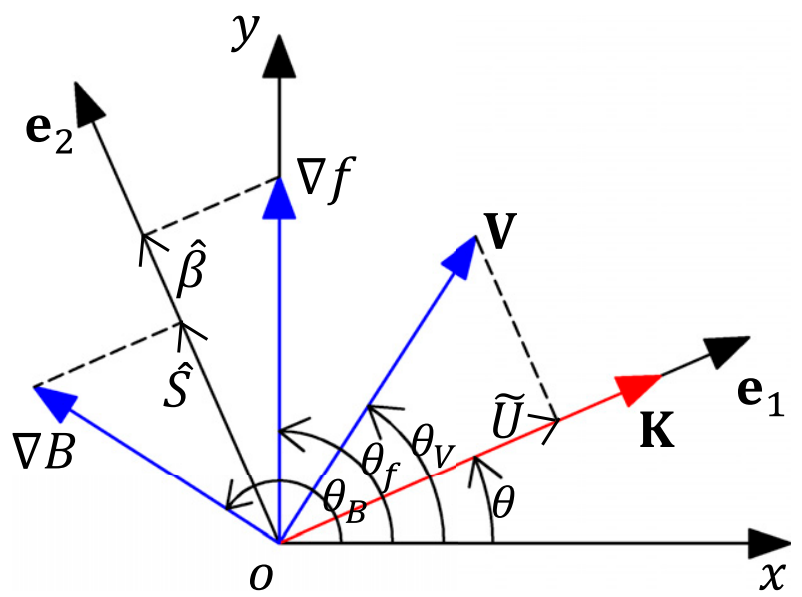

FIG. 2. Schematic of the wavenumber coordinate system. The wave vector $\mathbf{K}$ (red arrow) crosses the $x$ axis at an arbitrary angle $\theta$. The unit vectors $\mathbf{e}_{1}$ and $\mathbf{e}_{2}$ are set separately in the tangential and normal direction of $\mathbf{K}$. The basic flow vector $\mathbf{V}$, planetary vorticity gradient $\nabla f$, and bottom slope $\nabla B$ are all marked by blue arrows. Parameter $\tilde{U}$ is the projection of $\mathbf{V}$ on $\mathbf{e}_{1}$, while $\hat{\beta}$ and $\hat{S}$ are the projections of $\nabla f$ and $\nabla B$ on $\mathbf{e}_{2}$, respectively.

Once $\Delta<0$, the phase speed can be written in a complex form: $c=\sigma / K=c_{r}+i c_{i}$, where

$$
c_{r}=\frac{K^{2}\left(K^{2}+2 F_{2}\right) \tilde{U}-\left(2 K^{2}+F_{1}+F_{2}\right) \hat{\beta}-\left(K^{2}+F_{1}\right) \hat{S}}{2 K^{2}\left(K^{2}+F_{1}+F_{2}\right)},
$$

and

$$
c_{i}= \pm \frac{(-\Delta)^{1 / 2}}{2 K^{2}\left(K^{2}+F_{1}+F_{2}\right)} .
$$

The corresponding unstable growth rate is

$$
\sigma_{i}=\frac{(-\Delta)^{1 / 2}}{2 K\left(K^{2}+F_{1}+F_{2}\right)} .
$$

Note that $c_{r}$ is a linear function of $\tilde{U}, \hat{\beta}$, and $\hat{S}$ for a given perturbation with the wavenumber $K$, while $c_{i}$ and $\sigma_{i}$ are composed of the nonlinear terms $\left(\tilde{U}^{2}, \hat{\beta}^{2}, \hat{S}^{2}, \tilde{U} \hat{\beta}\right.$, $\tilde{U} \hat{S}$, and $\hat{\beta} \hat{S})$. Aside from the terms associated with $\hat{S}$, Eqs. (3.6a) and (3.6b) are precisely the same as the results of Pedlosky (1987, Eq. 7.13.11).

\section{Bottom slope effects on propagation and scale of unstable waves}

\section{a. Numerical case study}

In this section, the solutions [Eqs. (3.6a)-(3.6c)] are applied in two typical cases in the Northern Hemisphere

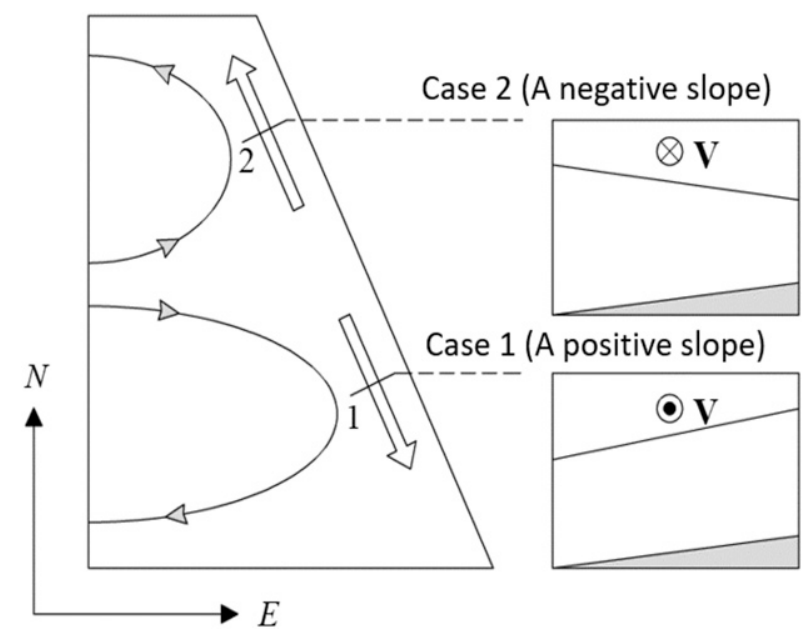

FIG. 3. Two typical flow/topography configurations. Case 1 is the "positive slope case" with the bottom slope in the same direction as the isopycnal tilt, which usually exists in the eastern boundary current of the subtropical gyre. Case 2 is the "negative slope case" with the bottom slope opposed to the isopycnal tilt, usually being found in the subpolar gyre.

(Fig. 3). The first one is the "positive slope case" with the bottom slope in the same direction as the isopycnal tilt, which usually exists in the eastern boundary current of the subtropical gyre (Fig. 3, Case 1); the second is the "negative slope case" with the bottom slope opposed to the isopycnal tilt, usually being found in the subpolar gyre (Fig. 3, Case 2). Parameters for the two cases are listed in Table 1 , where $L_{D}=\left(g^{\prime} H_{1}\right)^{1 / 2} / f_{0}$ is the first baroclinic Rossby deformation radius.

The growth rate of an unstable wave can be seen as a function of the wavenumber if the parameters are prescribed [according to Eq. (3.6c)]. Figure 4 shows the growth rate for the two specific cases in the wavenumber space (the positive slope case on the left and the negative slope case on the right), where $K$ is the distance to the origin and $\theta$ is the angle around the $x$ axis. The scale of a perturbation is $L=1 / K$. The basic flow is unstable only if the initial wavenumber vector falls into the color-filled instability domain. Extreme points marked by dark stars in Fig. 4 represent the most unstable modes, with the maximum growth rate $\sigma_{i m}$, the corresponding wavenumber $\left(K_{m}, \theta_{m}\right)$, and the instability scale $L_{m}=1 / K_{m}$.

\section{1) Short And LONG WAVE CUTOFF}

Each plot in Fig. 4 shows a short wavelength cutoff to the instability: any initial perturbation with its scale much smaller than $L_{D}$ always remains stable. The phenomenon can be seen as an artifact of the two-layer model. For large wavenumbers, the vertical scale of the 
TABLE 1. Parameters for the two cases.

\begin{tabular}{lcc}
\hline \hline Parameter & $\begin{array}{c}\text { Case } 1 \\
\text { (positive slope) }\end{array}$ & $\begin{array}{c}\text { Case } 2 \\
\text { (negative slope) }\end{array}$ \\
\hline$f_{0}\left(\mathrm{~s}^{-1}\right)$ & $7.27 \times 10^{-5}$ & $7.27 \times 10^{-5}$ \\
$\beta\left(\mathrm{m}^{-1} \mathrm{~s}^{-1}\right)$ & $1.98 \times 10^{-11}$ & $1.98 \times 10^{-11}$ \\
$H_{1}(\mathrm{~m})$ & 500 & 500 \\
$H_{2}(\mathrm{~m})$ & 2000 & 2000 \\
$g^{\prime}\left(\mathrm{m} \mathrm{s}^{-2}\right)$ & 0.01 & 0.01 \\
$F_{1}\left(\mathrm{~m}^{-2}\right)$ & $1.06 \times 10^{-9}$ & $1.06 \times 10^{-9}$ \\
$F_{2}\left(\mathrm{~m}^{-2}\right)$ & $2.65 \times 10^{-8}$ & $2.65 \times 10^{-8}$ \\
$L_{D}\left(\mathrm{~km}^{-2}\right.$ & 30.76 & 30.76 \\
$|\mathbf{V}|(\mathrm{m} \mathrm{s})$ & 0.20 & 0.20 \\
$\theta_{V}\left({ }^{\circ}\right)$ & 315 & 135 \\
$\theta_{B}\left({ }^{\circ}\right)$ & 45 & 45 \\
\hline
\end{tabular}

perturbation shrinks and when it can no longer be resolved by the two-layer model the instability is lost, while it is maintained in the continuously stratified model (e.g., the Charney model) for all wavenumbers. Bretherton (1966) verified the presence of the short wave cutoff in the two-layer model and provided a qualitative description of how the PV gradient destabilizes the short waves in the continuous model.

$$
l_{c}=2^{-1 / 2}\left\{\left[\left(\frac{\beta}{F_{1}|\mathbf{V}|}\right)^{2}+\frac{j^{4} \pi^{4}}{D^{4}}\right]^{1 / 2}-\frac{j^{2} \pi^{2}}{D^{2}}\right\}^{1 / 2}, \quad(j=1,2,3, \ldots,)
$$

where $\beta /\left(F_{1}|\mathbf{V}|\right)$ is a ratio of the meridional PV gradient $\beta$ to the zonal $\mathrm{PV}$ gradient $F_{1}|\mathbf{V}| ; D$ is the channel width, and $j \pi / D$ is the cross-channel wavenumber. For comparison, we consider a similar case-a northward basic current flowing over a flat bottom [the terms associated with the bottom slope in Eq. (3.5) vanish]. The critical wavenumber $K_{0}$ (dimensional) for the long wave cutoff is obtained by solving $\Delta=0$,

$$
K_{0}=2^{1 / 4} F_{1}^{1 / 2}\left\{1-\left[1-\left(\frac{\beta \cot \theta}{F_{1}|\mathbf{V}|}\right)^{2}\right]^{1 / 2}\right\}^{1 / 4} .
$$

Equation (4.2) differs from Eq. (4.1) in the dependence of the critical wavenumber on the wave direction $\theta$ rather than the cross-channel wavenumber $j \pi / D$. Of special interest is the case of $\theta=90^{\circ}$ (or $270^{\circ}$ ), where $K_{0}$ reduces to $0(\cot \theta=0)$. This suggests that the stabilizing effect of $\beta$ would be annulled if the wave vector is in the $y$ direction. For $l_{c}$, however, the corresponding wave vector must have a component in the $x$ direction since the cross-channel wavenumber satisfies $j \pi / D>0(j=1,2,3, \ldots$,$) , and consequently$
Blumsack and Gierasch (1972) captured a long wavelength cutoff by introducing a uniform sloping bottom into the Eady model. It is also reproduced in the two-layer model (second row of Fig. 4). The maximum critical perturbation scale, occurring at the inner circle, is approximately $2.5 L_{D}$ for case 1 and $2 L_{D}$ for case 2 . In a fixed direction $\theta=135^{\circ}$ (parallel to the basic flow streamlines), the growth rate is shown in Fig. 5 as a function of the wavenumber and bottom slope. The $f$-plane case (Fig. 5a) reveals striking similarities with the result of Blumsack and Gierasch (1972). Specifically, long waves become stable when a bottom slope is introduced. As the slope becomes more negative, unstable modes are confined in a narrower band and shift to higher wavenumbers. In Fig. 5b, $\beta$ does add an interesting effect that the long wave cutoff still exists even over a flat bottom (slope $=0$ ).

An analytical expression for the long wave cutoff induced by $\beta$ was first obtained by Walker and Pedlosky (2002). In their model (meridional currents in a northsouth channel; $\left.H_{1} / H_{2}=F_{2} / F_{1}=1\right)$, the dimensionless along-channel wavenumber $l_{c}$ for the long wave cutoff is of the form

the presence of $\beta$ would lead to the long wave cutoff. Critical wavenumber $K_{0}$ is identical to $l_{c}$ in describing the effect of $\beta$; both of them shift to higher wavenumbers as the ratio $\beta /\left(F_{1}|\mathbf{V}|\right)$ increases. Figure $5 \mathrm{c}$ presents the relation curve (solid) between $K_{0} / F_{1}^{1 / 2}$ and $\beta /\left(F_{1}|\mathbf{V}|\right.$ ) for different wave directions, along with a curve (dashed) for $l_{c}(j=1)$. As the wave vector deviates from the $y$ direction $\left(\theta\right.$ changes from $75^{\circ}$ to $\left.45^{\circ}\right)$, the relation curve becomes steeper. Typically, when $\theta=60^{\circ}$, the curve for $K_{0} / F_{1}^{1 / 2}$ is close to that for $l_{c}$.

\section{2) INSTABILITY SCALE}

On a sloping bottom, the instability domains for the two cases are in the shape of a donut due to the short and long wavelength cutoff (second row of Fig. 4). Obviously, the left donut is smaller and narrower than the right one. This difference in size is also found when the bottom slope and $\beta$ are considered simultaneously (fourth row of Fig. 4), whereas on a flat bottom, the instability domains for the two cases are of the same size (first and third row of Fig. 4). The results indicate that the bottom slope is the dominant factor in the instability scale. 


\section{Case 1 (positive slope)}

Case 2 (negative slope)
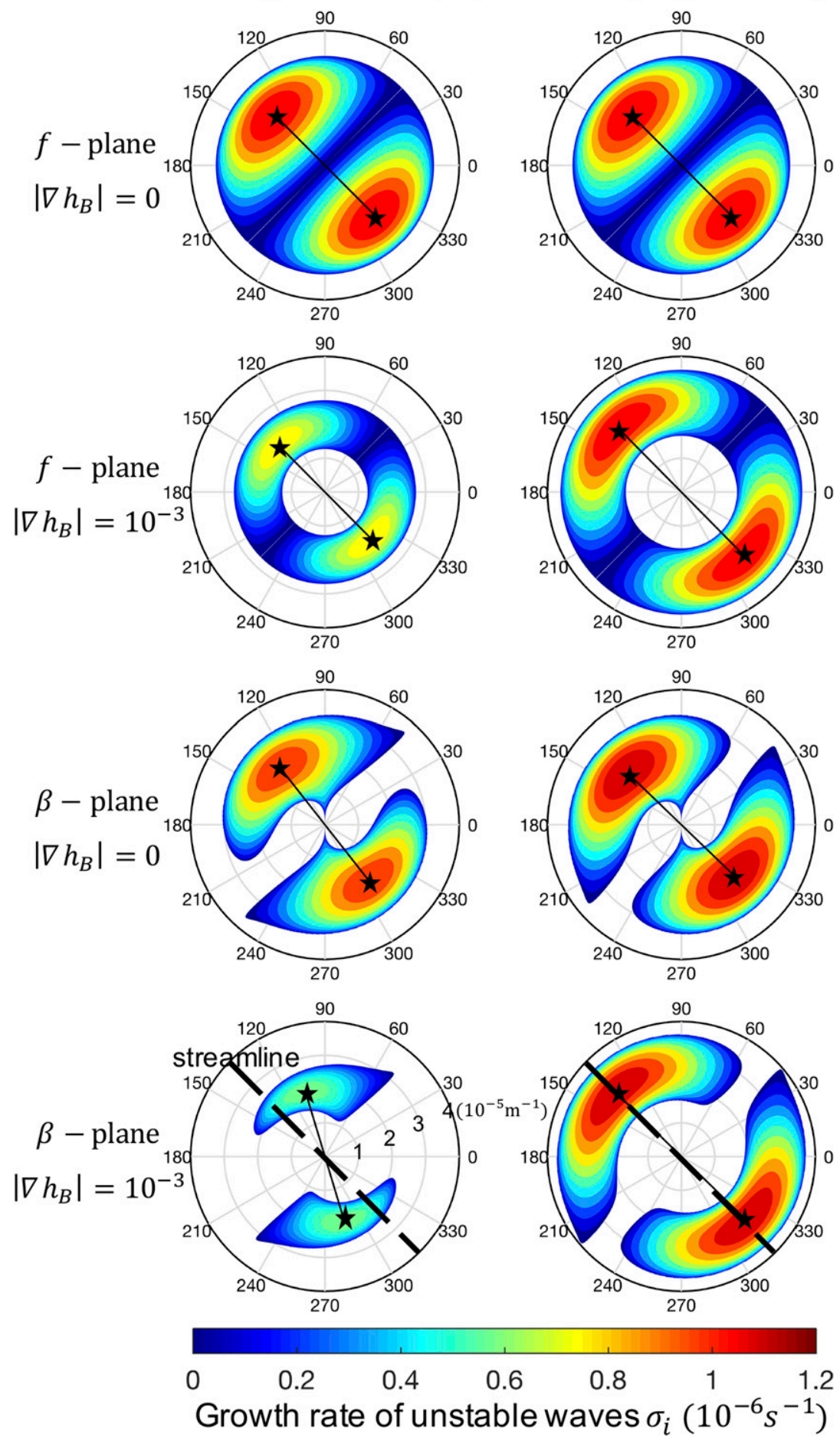

FIG. 4. Growth rate of unstable waves as a function of wavenumber for the two cases with a uniform basic velocity $|\mathbf{V}|=0.2 \mathrm{~m} \mathrm{~s}^{-1}$ : (left) the positive slope case and (right) the negative slope case. The black stars marked in each plot represent the most unstable modes. The dashed black streamline (in the last row) extends along the sloping bottom. 

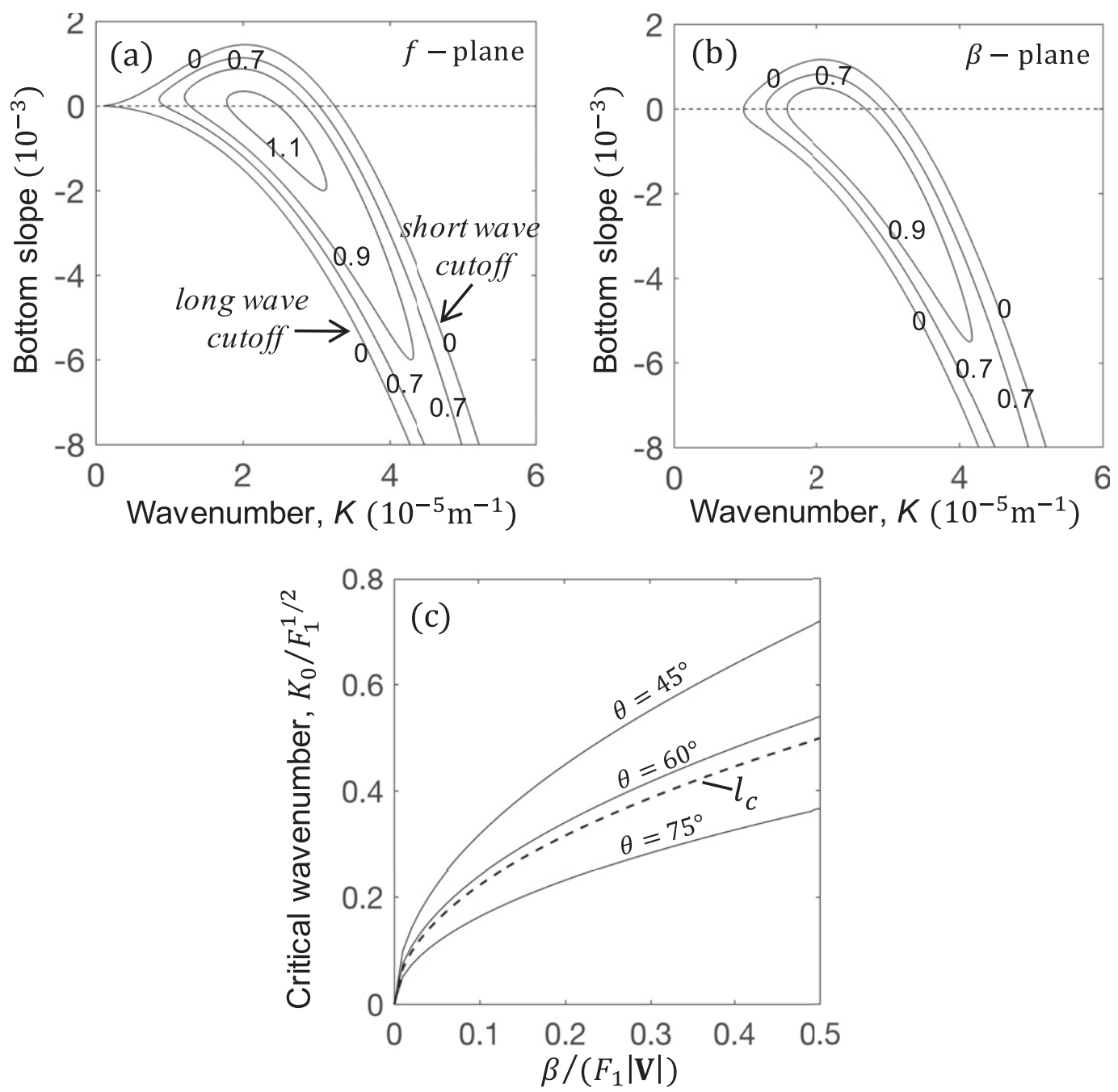

FIG. 5. Growth rate of unstable waves $\sigma_{i}\left(10^{-6} \mathrm{~s}^{-1}\right)$ as a function of wavenumber and bottom slope: (a) on the $f$ plane and (b) on the $\beta$ plane. (c) Critical wavenumber $K_{0} / F_{1}^{1 / 2}$ for the long wave cutoff as a function of $\beta /\left(F_{1}|\mathbf{V}|\right)$, with different wave directions $\left(45^{\circ}, 60^{\circ}, 75^{\circ}\right)$; the dashed curve describes the first mode of $l_{c}(j=1)$ for $D=100$ (Walker and Pedlosky 2002).

Figure 6 shows the relationship between the instability scale and bottom slope on the $f$ and $\beta$ planes. In the positive slope case (Fig. 6a), $L_{m}$ first increases, then decreases with slope. The change of $L_{m}$ is approximately $5 \mathrm{~km}$. Compared with the solid curve ( $f$ plane), the dashed curve ( $\beta$ plane) appears to be gentler, corresponding to a greater critical slope as well. In the negative slope case (Fig. 6b), the two curves are quite similar. Whether or not the $\beta$ effect is accounted for, the bottom slope effectively reduces the instability scale. From this perspective, the positive slope favors the unstable modes with lower wavenumbers (larger scales), while the negative slope favors those with higher wavenumbers (smaller scales). These results about the slope effects on the instability scale are in accordance with the previous studies based on zonal basic flows (Ikeda 1983; Wang and Ikeda 1997; Chen and Kamenkovich 2013).

\section{3) Propagation direction of the most UNSTABLE WAVE}

Although the most unstable wave vector must have a component parallel to the basic flow direction to produce an energy-releasing cross-current perturbation, the stabilizing effect of $\beta$ will tend to produce a component perpendicular to the flow. Then the question is, what is the role of the bottom slope in the propagation of the most unstable wave? As shown in the last row of Fig. 4, the most unstable modes (marked by dark stars) in the positive slope case deviate significantly from the 

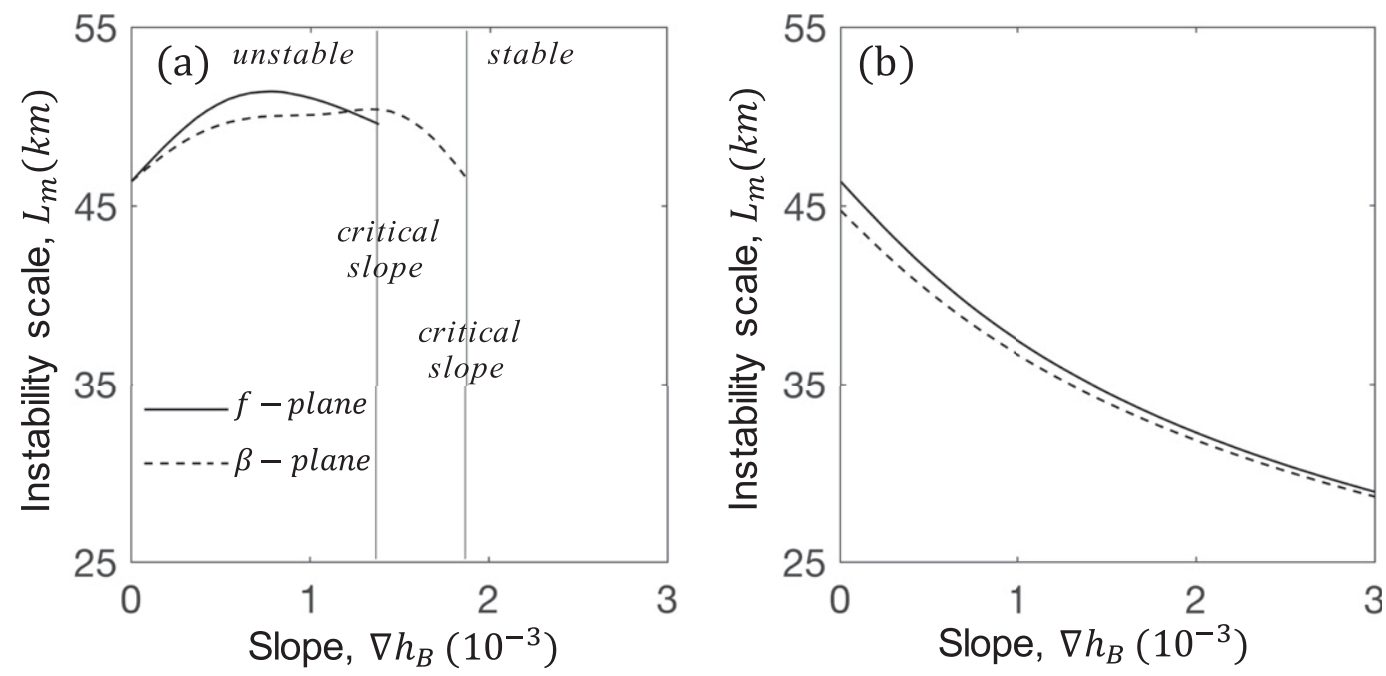

FIG. 6. The spatial scale of the most unstable wave as a function of bottom slope on the $f$ plane (solid curve) and $\beta$ plane (dashed curve): (a) for the positive slope case and (b) for the negative slope case.

streamline of basic flow, while in the negative slope case, the most unstable modes are distributed along the streamline.

The phase speed vector (the real part only) of the most unstable wave is given by $\mathbf{c}_{m}=c_{m} \mathbf{K}_{m} / K_{m}$, the orientation of which depends on the sign of $c_{m}$,

$$
\theta_{C_{m}}=\theta_{m} \pm \Gamma \pi, \quad \theta_{C_{m}} \in[0,2 \pi),
$$

where $\Gamma=0\left(c_{m} \geq 0\right)$ and $\Gamma=1\left(c_{m}<0\right)$.

Figure 7 presents the direction $\theta_{C_{m}}$ and phase speed $c_{m}$ of the most unstable wave as functions of the bottom slope. In the negative slope case (Fig. 7c), $\theta_{C_{m}}$ is not sensitive to the change of the steepness, and always closes to the basic flow direction $\left(\left|\theta_{C_{m}}-\theta_{V}\right|<0.02^{\circ}\right)$. However, in the positive slope case (Figs. 7a,b), both direction and phase speed of the most unstable wave are dramatically influenced by the steepness. The wave keeps propagating along the basic flow at a fairly high phase velocity $\left(3.8 \mathrm{~cm} \mathrm{~s}^{-1}\right)$ over a flat bottom, while it has to veer toward the normal direction of basic flow when the steepness increases. In the whole course, the angle deviation remains acute $\left(\left|\theta_{C_{m}}-\theta_{V}\right|<90^{\circ}\right)$ and the speed reaches the minimum $0.4 \mathrm{~cm} \mathrm{~s}^{-1}$ at a slope of $1.1 \times 10^{-3}$. Thus, the most unstable wave is likely to propagate across the streamline, heading to the shelf or deep basin.

\section{b. Theoretical analysis}

\section{1) Analytical solutions to the Angle DEVIATION}

To understand the propagation characteristics of the most unstable wave in the two cases, a theoretical analysis is presented in this section. In the reducedgravity model, the basic shear flow is balanced by the thermocline depth gradient,

$$
(-U, V)=\frac{g^{\prime}}{f_{0}} \nabla h,
$$

where $h$ is the spatially variable depth of the upper layer (see Fig. 1). With the setting $I=f_{0} h / H_{1}$, the complete expressions of the background PV gradient in the two layers are $\nabla Q_{1}=-\nabla I+\nabla f$ and $\nabla Q_{2}=\delta \nabla I+\nabla f+\nabla B$, respectively.

Two nondimensional numbers are introduced to reflect the effect of $\nabla f$ and $\nabla B$ relative to $\nabla I$,

$$
\begin{aligned}
& \varepsilon_{1}=\frac{|\nabla f|}{|\nabla I|}=\frac{\beta H_{1}}{f_{0}|\nabla h|}, \\
& \varepsilon_{2}=\frac{|\nabla B|}{|\nabla I|}=\delta \frac{\left|\nabla h_{B}\right|}{|\nabla h|} .
\end{aligned}
$$

Using the parameter values in Table $1(|\nabla f| \approx$ $2 \times 10^{-11} \mathrm{~m}^{-1} \mathrm{~s}^{-1}$ and $\left.|\nabla I| \approx 2 \times 10^{-10} \mathrm{~m}^{-1} \mathrm{~s}^{-1}\right), \varepsilon_{1}$ is of the order of $10^{-1}$. Parameter $\varepsilon_{2}$ changes with the steepness. Particularly when $\left|\nabla h_{B}\right|$ is comparable with $|\nabla h|\left(=1.45 \times 10^{-3}\right), \varepsilon_{2}$ will be roughly equivalent to $\delta\left(=H_{1} / H_{2}=0.25\right)$.

Now, we take the purely meridional current as an extreme case of nonzonality and give the analytic expression of the angle deviation $\Delta \theta=$ $\left|\theta_{C_{m}}-\theta_{V}\right|\left(\theta_{V}\right.$, the basic flow direction; $\theta_{C_{m}}$, the most unstable wave direction; see the appendix for the full derivation), 

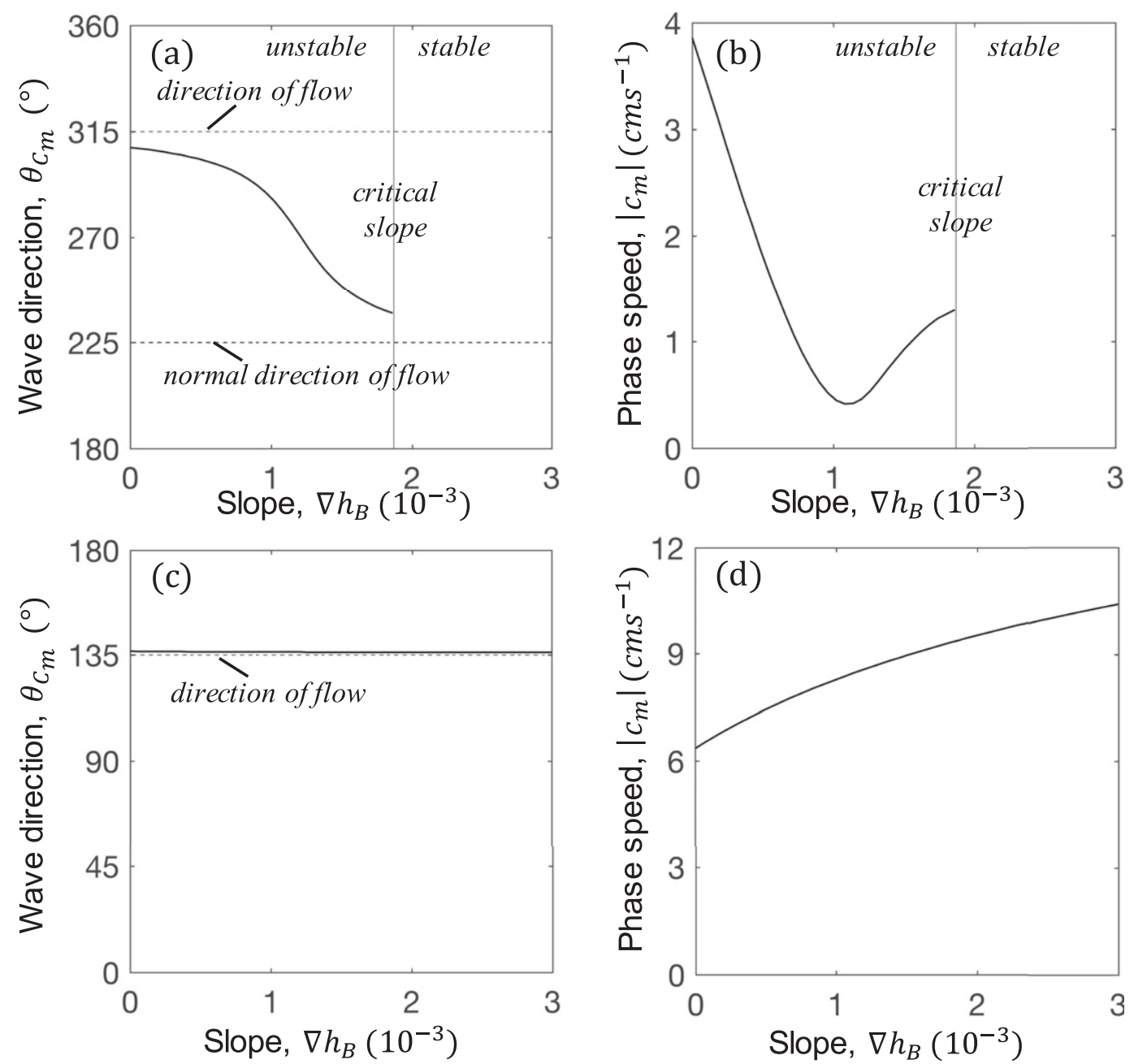

FIG. 7. Phase speed and direction of the most unstable wave as functions of bottom slope on the $\beta$ plane: (a),(b) for the positive slope case and (c),(d) for the negative slope case.

$\Delta \theta=\frac{1}{2}\left|\tan ^{-1}\left[\frac{\varepsilon_{1}\left(M_{4}-\varepsilon_{2} M_{6}\right)}{M_{1}-\varepsilon_{1}^{2} M_{2}+\varepsilon_{2}^{2} M_{3}-\varepsilon_{2} M_{5}}\right]\right|$,

for the positive slope case, and

$\Delta \theta=\frac{1}{2}\left|\tan ^{-1}\left[\frac{\varepsilon_{1}\left(M_{4}+\varepsilon_{2} M_{6}\right)}{-M_{1}+\varepsilon_{1}^{2} M_{2}-\varepsilon_{2}^{2} M_{3}-\varepsilon_{2} M_{5}}\right]\right|$,

for the negative slope case, where $M_{1}=-r^{2}\left(4 \delta-r^{2}\right)$, $M_{2}=(1+\delta)^{2}, M_{3}=(1+r)^{2}, M_{4}=-2 r^{2}(1-\delta), M_{5}=$ $-2 r\left(r^{2}+r-2 \delta\right)$, and $M_{6}=2 r(1-\delta)+1+\delta^{2}$. The parameter $r$ defined as $r=K^{2} / F_{1}=L_{D}^{2} / L^{2}$ describes the scale of the deformation radius relative to the perturbation; $\delta=H_{1} / H_{2}$ is a given constant.

According to Eqs. (4.6a) and (4.6b), $\Delta \theta$ will vanish in the condition of $\varepsilon_{1}=0(|\nabla f|=0)$. Thus, it is appropriate to discuss the propagating direction on the $\beta$ plane $\left(\varepsilon_{1}=10^{-1}\right)$. As depicted in Fig. 8a, the angle deviation becomes more pronounced when the positive slope increases. The result is in agreement with the case study (Fig. 7a). It is also found that the tilt of the relation curve is sensitive to the selection of $r$. Given $L_{D}=30 \mathrm{~km}$, the scale $L_{m}$ is approximately $50 \mathrm{~km}$, and then $r$ for the most unstable wave is 0.36 . Thus, the curve for $r_{m}=0.36$ in Fig. 8a describes the dependence of the angle deviation of the most unstable wave on the bottom slope. In the negative slope case (Fig. 8b), the angle deviation is confined to a narrow range, especially for the wave with a relatively larger scale (smaller $r$ ).

\section{2) MECHANISM FOR BOTTOM SLOPE EFFECTS ON PROPAGATION}

Baroclinic instability can be regarded as a selfreinforcing process that relies on the interaction of the 

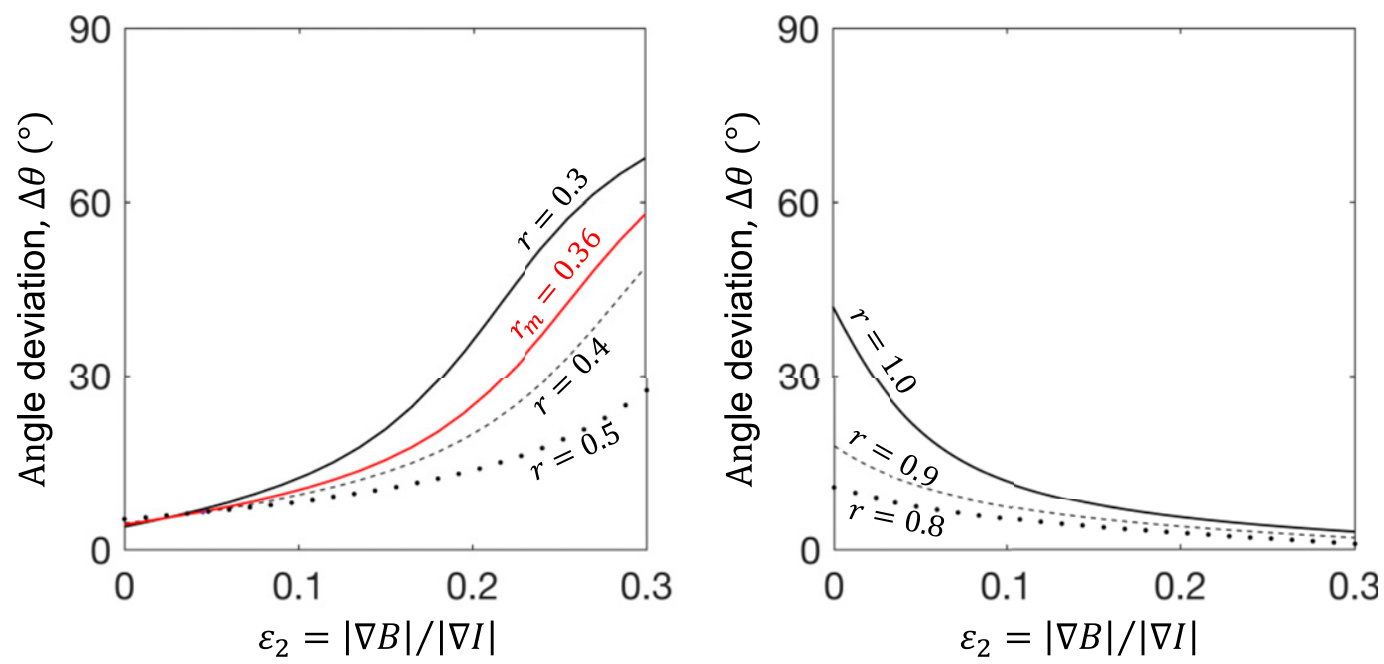

FIG. 8. The angle deviation as a function of $\varepsilon_{2}(=|\nabla B| /|\nabla I|)$, with various $r\left(=K^{2} / F_{1}=L_{D}^{2} / L^{2}\right)$ : (a) for the positive slope case and (b) for the negative slope case. Given $L_{D}=30 \mathrm{~km}$, the curve for $r_{m}=0.36$ in the positive slope case describes the dependence of the angle deviation of the most unstable wave on the bottom slope.

motions in the two layers. The vortex tubes of the upper and lower layer combine to release available potential energy in a way that reduces the interface slope (Cushman-Roisin and Beckers 2011). A bottom slope can effectively influence the instability process by modifying the background PV gradient of the lower layer and therefore alter the phase speed direction of unstable perturbations.

The fields of background PV in each layer for the southward flow case, with or without a bottom slope, are shown in Figs. 9a and 9b, respectively. Given the condition of $\mathbf{c}_{m} \cdot \mathbf{V}>0$, the unstable wave must travel downstream rather than propagate against the basic current. For the upper layer, the PV gradient $\left(\nabla Q_{1}=-\nabla I+\nabla f\right)$ almost points east due to $\varepsilon_{1}=$ $|\nabla f| /|\nabla I|=10^{-1}$, and the unstable wave prefers to travel southward along the contours of PV for extracting as much energy as possible. With a flat bottom, the PV contours of the lower layer are roughly meridional $\left(\nabla Q_{2}=\delta \nabla I+\nabla f\right.$ points west). According to the form of the traveling wave solution in Eq. (3.1), perturbations within the two layers should not propagate in opposite directions, which suggests a nearly southwardpropagating wave in the lower layer (Fig. 9a). Ultimately, the coupling effect of the two layers favors the wave with the maximum growth rate propagating along the basic flow direction.

A positive slope is introduced in Fig. 9b, where the zonal PV gradient of the lower layer is reduced by the slope so that the unstable wave chooses a southwest route parallel to the modified PV contours. Considering the interaction of the two layers, there will be a tendency for the most unstable wave to propagate across the basic flow.

In the case of northward flow as depicted in Figs. 9c and $9 \mathrm{~d}$, with or without a bottom slope, the PV contours in each layer generally extend along the meridian. This result should be attributed to the slope's positive contribution to zonal PV gradient, which puts $\nabla f$ in a position of less importance. Therefore, in the negative slope case, the most unstable wave always propagates along the streamlines.

\section{Conclusions and discussion}

A modified two-layer model has been employed to investigate the instability of nonzonal baroclinic currents flowing over a sloping bottom. The model results are in conformity with earlier work in certain respects, such as short/long wave cutoff and instability scale. In particular, we studied the bottom slope effects on the propagation of the most unstable wave. The conclusions are summarized as follows.

1) The case study suggests two kinds of long wave cutoff: the first one is induced by the bottom slope and exists in all directions, and the second is produced by $\beta$, which requires at least a slight deviation of the wave vector from the meridian. Along with the increases of the negative slope and $\beta$, the minimum critical wavenumber shifts to higher values. A short wave cutoff is always present, and the corresponding critical scale is of the order of the Rossby deformation radius. 


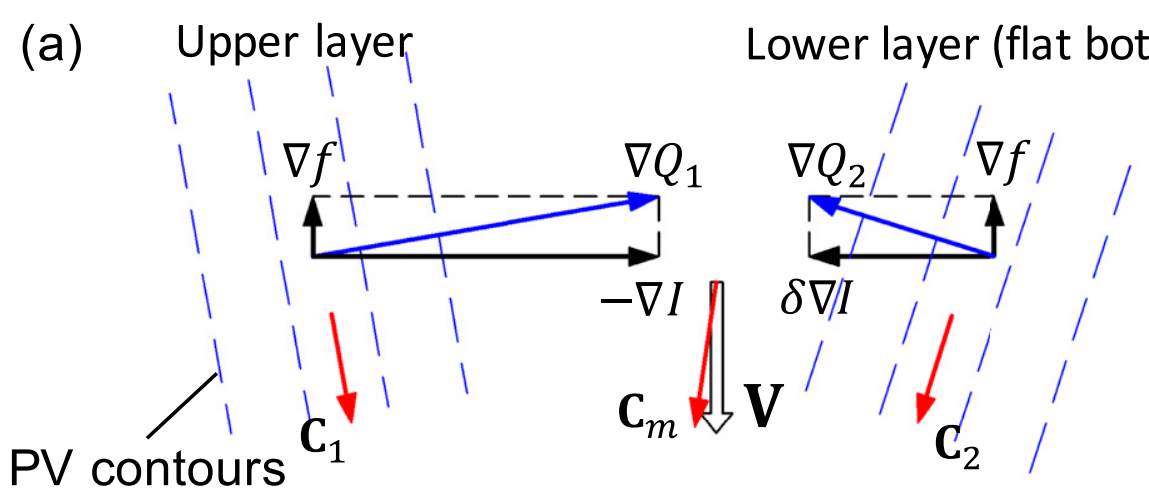

(b) Upper layer

Lower layer (a positive slope)
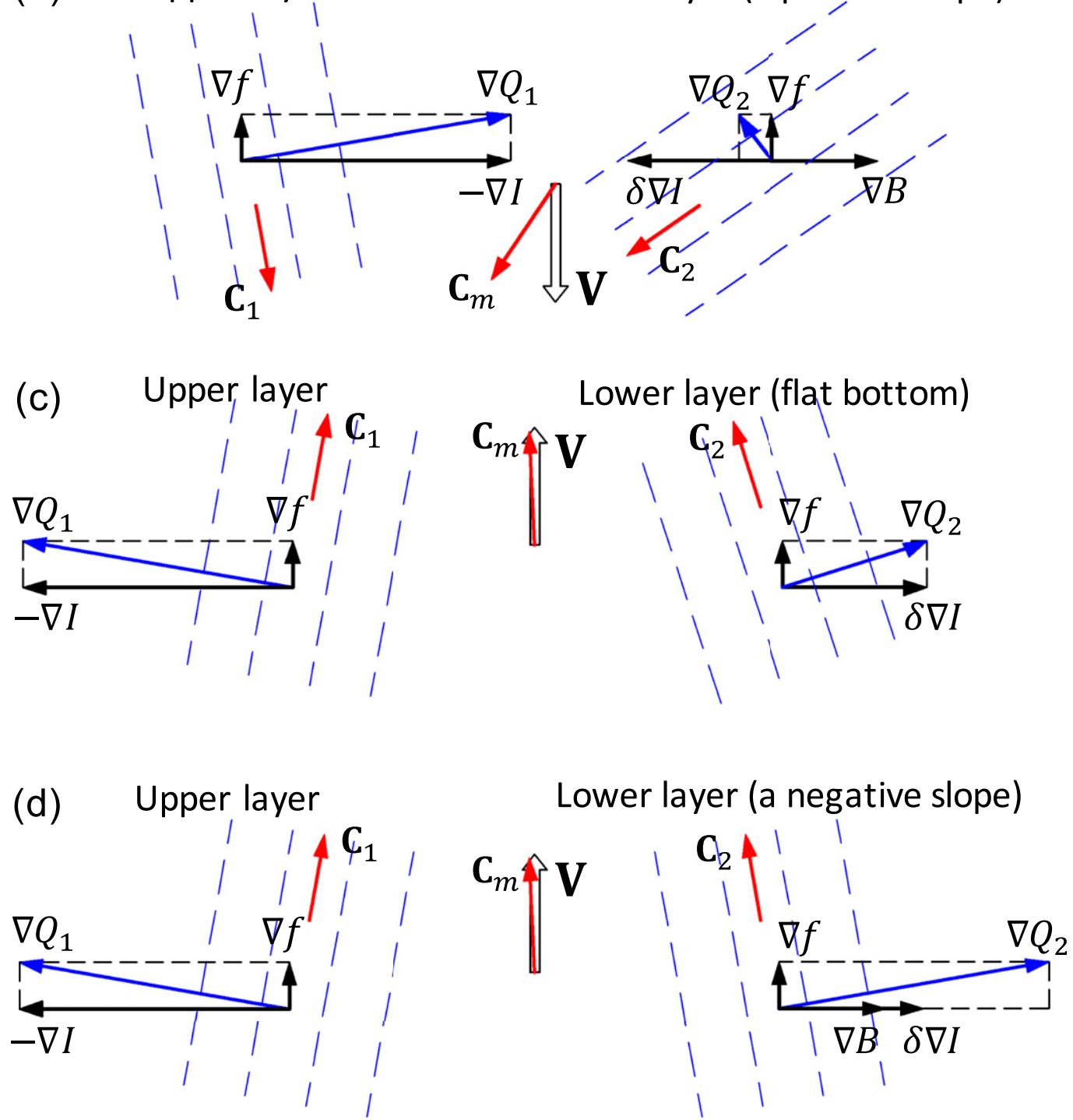

FIG. 9. Schematic diagram for describing the bottom slope effect on the propagation of the most unstable wave: (a),(b) for a southward flow (the positive slope case) and (c),(d) for a northward flow (the negative slope case). The complete expressions of the PV gradient in the two layers are $\nabla Q_{1}=-\nabla I+\nabla f$ and $\nabla Q_{2}=\delta \nabla I+\nabla f+\nabla B$. 
2) The background PV gradient consists of three parts: thermocline depth gradient $\nabla I$, planetary vorticity gradient $\nabla f(\beta)$, and bottom slope $\nabla B$. The deviation of the most unstable wave from the basic flow streamlines is determined by two indices: $\varepsilon_{1}=|\nabla f| /|\nabla I|$ and $\varepsilon_{2}=|\nabla B| /|\nabla I|$. Only when the planetary vorticity gradient is taken into account $\left(\varepsilon_{1} \neq 0\right)$ would a bottom slope play a role in altering the propagation direction of the most unstable wave.

3) The bottom slope, indeed, plays distinct roles in the two typical flow/topography cases. For case 1 , a positive slope will help the fastest-growing wave propagate across background flows by modifying the PV gradient of the lower layer via weakening or even cancelling the effect of $\nabla I$, and thus making the $\beta$ effect more important. For case 2 , the effect of $\nabla I$, which has the same direction as $\nabla B$, is strengthened by a negative slope. Consequently, the $\beta$ effect appears negligible and the most unstable wave almost propagates along the basic flows.

4) A bottom slope can also change the instability scale. For the positive slope case, the scale increases first, and then decreases with slope. While for the negative slope case, whether or not the $\beta$ effect is considered, the bottom slope effectively reduces the instability scale.

Our findings about bottom slope effects on the propagation of the most unstable wave should be useful to help us understand the track of mesoscale eddies in the ocean. To our minds, a tendency to propagate across the basic currents at the initial time provides a precondition for the eddy to ride on Rossby waves and move to the interior. For example, the eddies generated near the California coast can travel considerable distances westward (Kurian et al. 2011), while those formed on the east coast of the Gulf of Alaska always propagate along the shore (Thomson and Gower 1998; Shore et al. 2008), which is consistent with the results of instability analysis. More detailed work including observations, simulations, and theoretical study remains to be conducted to prove this point. Before that, this paper puts forward some tentative ideas about the slope effects.

Acknowledgments. The authors thank two anonymous reviewers. This work was supported by grants from National Key Research and Development Program of China (Grant 2017YFA0604600), National Natural Science Foundation of China (Grant 41676019) and the Fundamental Research Funds for the Central Universities.

\section{APPENDIX}

\section{The Derivation of the Angle between the Most Unstable Wave and Basic Flow}

Substitution of Eq. (4.4) into Eq. (3.3a) yields

$$
\tilde{U}=-\frac{1}{F_{1}} \hat{I}
$$

and

$$
\hat{I}=\frac{\mathbf{K} \times \nabla I}{K}=-|\nabla I| \cos \left(\theta_{V}-\theta\right),
$$

the projection of $\nabla I$ on the normal direction of the wave vector.

Consider the basic currents flowing along the bottom slope, $\theta_{B}$ should satisfy

$$
\theta_{B}=\theta_{V}+\frac{(-1)^{j}}{2} \pi
$$

where $j=0$ for the positive slope case, and $j=1$ for the negative slope case.

With Eqs. (A.1), (A.2), and (A.3), Eq. (3.5) can be rewritten in a polar form,

$$
\begin{aligned}
\Delta= & F_{1}^{2}\left[M_{1}|\nabla I|^{2} \cos ^{2}\left(\theta_{V}-\theta\right)+M_{2}|\nabla f|^{2} \cos ^{2} \theta+M_{3}|\nabla B|^{2} \cos ^{2}\left(\theta_{V}-\theta\right)\right. \\
& -M_{4}|\nabla I||\nabla f| \cos \left(\theta_{V}-\theta\right) \cos \theta-(-1)^{j} M_{5}|\nabla I||\nabla B| \cos ^{2}\left(\theta_{V}-\theta\right) \\
& \left.+(-1)^{j} M_{6}|\nabla f||\nabla B| \cos \left(\theta_{V}-\theta\right) \cos \theta\right] .
\end{aligned}
$$

First, special attention is required for the perturbation that propagates in a direction perpendicular to the basic flow (symmetric perturbation), for which the terms associated with $\cos \left(\theta_{V}-\theta\right)$ will reduce to zero so that
$\Delta=F_{1}^{2} M_{2}|\nabla f|^{2} \cos ^{2} \theta \geq 0$. Thus, for the most unstable mode, $\theta_{C_{m}}$ must satisfy

$$
\left|\theta_{C_{m}}-\theta_{V}\right| \neq \pi / 2,3 \pi / 2 .
$$


Another constraint for $\theta_{C_{m}}$ is given by

$$
\mathbf{c}_{m} \cdot \mathbf{V}=\frac{K_{m}^{2}\left(K_{m}^{2}+2 F_{2}\right) \tilde{U}^{2}-\left(2 K_{m}^{2}+F_{1}+F_{2}\right) \tilde{U} \hat{\beta}-\left(K_{m}^{2}+F_{1}\right) \tilde{U} \hat{S}}{2 K_{m}^{2}\left(K_{m}^{2}+F_{1}+F_{2}\right)}>0
$$

which is valid so long as the basic shear flow is strong enough. This condition implies a tendency for $\mathbf{c}_{m}$ to be in the same direction as or cross $\mathbf{V}$ at an acute angle, namely,

$$
\left|\theta_{C_{m}}-\theta_{V}\right| \in[0, \pi / 2) .
$$

According to Eq. (3.6c), $\sigma_{i}$ and $\Delta$ share the same extreme value point $\theta_{m}$, and therefore $\theta_{m}$ can be identified by solving $\partial \Delta / \partial \theta=0$.

From (A.4), $\partial \Delta / \partial \theta$ is obtained,
For the positive slope case, $j=0\left(\theta_{V}=3 \pi / 2, \theta_{B}=0\right)$, Eq. (A.8) reduces to

$$
\frac{\partial \Delta}{\partial \theta}=\lambda \sin (2 \theta+\mu)
$$

$$
\begin{aligned}
\frac{\partial \Delta}{\partial \theta}= & F_{1}^{2}\left\{\left[M_{1}|\nabla I|^{2}+M_{3}|\nabla B|^{2}-(-1)^{j} M_{5}|\nabla I||\nabla B|\right] \sin \left(2 \theta_{V}-2 \theta\right)+\left[-M_{4}|\nabla I||\nabla f|\right.\right. \\
& \left.\left.+(-1)^{j} M_{6}|\nabla f||\nabla B|\right] \sin \left(\theta_{V}-2 \theta\right)-M_{2}|\nabla f|^{2} \sin (2 \theta)\right\} .
\end{aligned}
$$

where

$$
\begin{aligned}
\lambda= & F_{1}^{2}\left[\left(M_{1}|\nabla I|^{2}-M_{2}|\nabla f|^{2}+M_{3}|\nabla B|^{2}-M_{5}|\nabla I||\nabla B|\right)^{2}\right. \\
& \left.+\left(M_{4}|\nabla I||\nabla f|-M_{6}|\nabla f||\nabla B|\right)^{2}\right]^{1 / 2},
\end{aligned}
$$

and

$$
\mu=\tan ^{-1}\left[\frac{M_{4}|\nabla I||\nabla f|-M_{6}|\nabla f||\nabla B|}{M_{1}|\nabla I|^{2}-M_{2}|\nabla f|^{2}+M_{3}|\nabla B|^{2}-M_{5}|\nabla I||\nabla B|}\right] .
$$

By solving $\partial \Delta / \partial \theta=0$ under the constraint of Eq. (A.5), $\theta_{m}$ is related to $\mu$,

$\theta_{m}=-\frac{\mu}{2} \pm \frac{N}{2} \pi, \quad\left(\theta_{m} \in[0,2 \pi), N=1,3,5, \ldots,\right)$,

from which, with Eqs. (4.3) and (A.10b) and the condition of Eq. (A.7), it follows that

$$
\Delta \theta=\left|-\frac{\mu}{2}\right|=\frac{1}{2}\left|\tan ^{-1}\left[\frac{\varepsilon_{1}\left(M_{4}-\varepsilon_{2} M_{6}\right)}{M_{1}-\varepsilon_{1}^{2} M_{2}+\varepsilon_{2}^{2} M_{3}-\varepsilon_{2} M_{5}}\right]\right|
$$

where $\Delta \theta=\left|\theta_{C_{m}}-\theta_{V}\right|$ is the angle deviation (the positive slope case).

Analogously, the application of the condition $j=1$ $\left(\theta_{V}=\pi / 2, \theta_{B}=0\right)$ into Eq. (A.8) yields the solution for the negative slope case,

$$
\Delta \theta=\left|-\frac{\mu}{2}\right|=\frac{1}{2}\left|\tan ^{-1}\left[\frac{\varepsilon_{1}\left(M_{4}+\varepsilon_{2} M_{6}\right)}{-M_{1}+\varepsilon_{1}^{2} M_{2}-\varepsilon_{2}^{2} M_{3}-\varepsilon_{2} M_{5}}\right]\right| .
$$

\section{REFERENCES}

Arbic, B. K., 2000: Generation of mid-ocean eddies: The local baroclinic instability hypothesis. Ph.D. dissertation, Massachusetts Institute of Technology-Woods Hole Oceanographic Institution Joint Program, 290 pp.

Blumsack, S. L., and P. J. Gierasch, 1972: Mars: The effects of topography on baroclinic instability. J. Atmos. Sci., 29, 10811089, https://doi.org/10.1175/1520-0469(1972)029<1081: MTEOTO > 2.0.CO;2.

Bretherton, F. P., 1966: Baroclinic instability and the short wavelength cut-off in terms of potential vorticity. Quart. J. Roy. Meteor. Soc., 92, 335-345, https://doi.org/10.1002/ qj.49709239303.

Charney, J. G., 1947: The dynamics of long waves in a baroclinic westerly current. J. Meteor., 4, 136-162, https://doi.org/10.1175/ 1520-0469(1947)004<0136:TDOLWI>2.0.CO;2. 
Chen, C., and I. Kamenkovich, 2013: Effects of topography on baroclinic instability. J. Phys. Oceanogr., 43, 790-804, https:// doi.org/10.1175/JPO-D-12-0145.1.

Cushman-Roisin, B. C., and J. Beckers, 2011: Instabilities of rotating stratified flows. Introduction to Geophysical Fluid Dynamics, B. Cushman-Roisin and J.-M. Beckers, Eds., International Geophysics, Vol. 101, Academic Press, 553-587, https://doi.org/10.1016/B978-0-12-088759-0.00017-1.

Eady, E. T., 1949: Long waves and cyclone waves. Tellus, 1 (3), 33-52, https://doi.org/10.3402/tellusa.v1i3.8507.

Gill, A. E., J. S. A. Green, and A. J. Simmons, 1974: Energy partition in the large-scale ocean circulation and the production of mid-ocean eddies. Deep-Sea Res. Oceanogr. Abstr., 21, 499 528, https://doi.org/10.1016/0011-7471(74)90010-2.

Hristova, H. G., J. Pedlosky, and M. A. Spall, 2008: Radiating instability of a meridional boundary current. J. Phys. Oceanogr., 38, 2294-2307, https://doi.org/10.1175/2008JPO3853.1.

Ikeda, M., 1983: Linear instability of a current flowing along a bottom slope using three-layer model. J. Phys. Oceanogr., 13, 208-223, https://doi.org/10.1175/1520-0485(1983)013<0208: LIOACF $>2.0 . \mathrm{CO} ; 2$.

Kamenkovich, I., and J. Pedlosky, 1996: Radiating instabilities of nonzonal ocean currents. J. Phys. Oceanogr., 26, 622-643, https://doi.org/10.1175/1520-0485(1996)026<0622:RIONOC> 2.0.CO;2.

Kurian, J., F. Colas, X. Capet, J. McWilliams, and D. Chelton, 2011: Eddy properties in the California Current System. J. Geophys. Res., 116, C08027, https://doi.org/10.1029/ 2010JC006895.

McWilliams, J. C., and Coauthors, 1983: The local dynamics of eddies in the western North Atlantic. Eddies in Marine Science, A. R. Robinson, Ed., Springer, 92-113 pp.

Mysak, L. A., 1977: On the stability of the California Undercurrent off Vancouver Island. J. Phys. Oceanogr., 7, 904-917, https://doi.org/10.1175/1520-0485(1977)007<0904:OTSOTC> 2.0.CO;2.

Orlanski, I., 1969: The influence of bottom topography on the stability of jets in a baroclinic fluid. J. Atmos. Sci., 26, 1216-1232, https://doi.org/10.1175/1520-0469(1969)026<1216: TIOBTO $>2.0 . \mathrm{CO} ; 2$.

Pedlosky, J., 1987: Instability theory. Geophysical Fluid Dynamics, 2nd ed., Springer, 490-623.
Phillips, N. A., 1954: Energy transformations and meridional circulations associated with simple baroclinic waves in a two-level quasi-geostrophic model. Tellus, 6, 273-286, https://doi.org/10.1111/j.2153-3490.1954.tb01123.x.

Rhines, P. B., 1977: The dynamics of unsteady currents. Marine Modeling, E. A. Goldberg et al., Eds., The Sea-Ideas and Observations on Progress in the Study of the Seas, Vol. 6, Wiley and Sons, 189-318.

Shore, J., M. W. Stacey, and D. G. Wright, 2008: Sources of eddy energy simulated by a model of the northeast Pacific Ocean. J. Phys. Oceanogr., 38, 2283-2293, https://doi.org/10.1175/ 2008JPO3800.1.

Smith, K. S., 2007: Eddy amplitudes in baroclinic turbulence driven by nonzonal mean flow: Shear dispersion of potential vorticity. J. Phys. Oceanogr., 37, 1037-1050, https://doi.org/ 10.1175/JPO3030.1.

Spall, M. A., 2000: Generation of strong mesoscale eddies by weak ocean gyres. J. Mar. Res., 58, 97-116, https://doi.org/10.1357/ 002224000321511214.

Steinsaltz, D., 1987: Instability of baroclinic waves with bottom slope. J. Phys. Oceanogr., 17, 2343-2350, https://doi.org/10.1175/ 1520-0485(1987)017<2343:IOBWWB >2.0.CO;2.

Tanaka, K., and K. Akitomo, 2001: Baroclinic instability of density current along a sloping bottom and the associated transport process. J. Geophys. Res., 106, 2621-2638, https://doi.org/ 10.1029/2000JC000214.

Thomson, R. E., and J. F. R. Gower, 1998: A basin-scale oceanic instability event in the Gulf of Alaska. J. Geophys. Res., 103, 3033-3040, https://doi.org/10.1029/97JC03220.

Walker, A., and J. Pedlosky, 2002: Instability of meridional baroclinic currents. J. Phys. Oceanogr., 32, 1075-1093, https:// doi.org/10.1175/1520-0485(2002)032<1075:IOMBC >2.0.CO;2.

Wang, J., and M. Ikeda, 1997: Diagnosing ocean unstable baroclinic waves and meanders using the quasigeostrophic equations and Q-vector method. J. Phys. Oceanogr., 27, 1158-1172, https://doi.org/10.1175/1520-0485(1997)027<1158:DOUBWA> 2.0.CO;2.

Xue, H., and G. L. Mellor, 1993: Instability of the Gulf Stream front in the South Atlantic Bight. J. Phys. Oceanogr., 23, 2326-2350, https://doi.org/10.1175/1520-0485(1993)023<2326:IOTGSF> 2.0.CO;2. 